\title{
Calcul des Barrages-Voûtes
}

\author{
I'ar Georges PRUDON, Inginieur, Protesseur à l'Institut Polytechnique de Grenoble
}

\begin{abstract}
Diffírentes mithodes de calcul, concernunt les barrages arqués, ont ité proposécs par les auteurs les plus qualifiés et les plus compétents. Wais tous s'accordent à reconnaitre que ce calcul est, en toute rigueur, absolument impossible.

Les uns admettont que la forme en arc ne joue qu'un rôle secondaire el que le barrage travaille surtont conme un mur rectiligne s'opposant par son poids à la poussée, c'est lancienne théorie classique.

D'attres, principalement en tmérique, considerent la poussée comme supportéc par des arcs horizontaux encastrés aux culées, le mur n'intervenant plus. Il est évident que le burrage arqué tranaille à la fois comme mur et comme are, mais la détermination exacte de la part de poussié supportétespectinement par le mur et par l'arc est parfatement impossible dans les cas pratiques.

Il faut remarquer d'ailleurs que les efforts thermiques produits par les variations de température produisent des efforts plus considérables que la poussér de leau. Donc loule détermination exacte devient illusoire.

Nous nous proposons dans cette note dexpliquer

$1^{0^{-}}$Cne mëthode qui serail parfaite si elle était d'une application moins ardue;

$2^{\circ}$ De donner une méthode noins exacte, mais beaucoup plus simple permettant de fixer approximativement le soulagement produit par la jorme en arc :

$3^{\circ} D^{\prime} e n$ déduire ce que devrail ètre le profil du barrage pour faire tramailler à la fois le mur et les arcs, profil correspondant au maximum d'économie.

Notre désir sera réalisé si les techniciens qualifiés en la malière veulent bien juger cette note dans son domaine qui est celui de la théorie et en déduire les conclusions praliques qu'elle mérite au non.
\end{abstract}

\section{MÉThode EXACTE}

cute cunsiscerait à décomposer le mur sur toute sa hauteur et l'arc suivant son développement en un certain nombre de parties égales.

La division du mur se faisant par des plans horizontaux, la division de l'arc par des plans normaux verticaux. Si on divise des deux façons en $n$ parties, le parement amont du barrage est découpé en $n^{2}$ surfaces élémentaires assimilables à des rectangles de surface $\omega$, la profondeur du centre de chaque rectangle au dessous de l'eau est $x$.

La poussée de l'eau sur le rectangle étant $p=x(1)$ une part: Kow revient au mur, la part $(1-K)$ wix revient à l'arc.

Le coefficient $K<1$, de poussée est d'ailleurs variable d'un élément à l'autre, et on voit de suite que le problème revient à calculer ce coefficient $K$ en tout point.

Nous négligerons dans toute cette étude l'influence des efforts tangentiels sur les déformations parce qu'elle est secondaire et que son introduction complique les calculs déjà peu simples.

Une façon (qui se présente à l'esprit de suite) de calculer les coefficients $K$ est d'exprimer que, en chaque point, la flèche prise par l'élément mur auquel appartient la surface élémentaire (1) est la même que la flèche prise par l'élément arc au même point.

Cette identification peut se faire, connaissant les lignes d'influence des flèches, suivant les éléments murs et suivant les éléments arcs. Une ligne d'influence d'un élément mur est facile à déterminer.

Considẻrons en effet (fig. 1) une tranche verticale de mur de largeur égale à 1 mètre ; appliquons à une section $S$ une charge normale au parement amont $\mathrm{P}=1$, le diagramme des moments fléchissants est une simple droite à $45^{\circ}$.

En appelant E le coefficient d'élasticité de la matière $(2.000$ klgs $\mathrm{m} / \mathrm{m}^{2}$ ), I le moment d'inertie variable de la section horizontale, on obtient la ligne d'influence des flèches en traçant; avec une distance polaire égale à $E$ un funiculaire des forces fictives $\frac{M \unlhd x}{I}$. Ce funiculaire C ayant d'ailleurs son côté de départ langent au parement amont en bas.
Rappelons ici que si la charge 1 est appliquée en S' elle produit en $\mathrm{S}$ une flèche mesurée par l'ordonnée $y$ ' de la ligne d'influence en S'c. Donc la ligne d'influence 'relative à $S$ ' passe par $A$. Cette propriété ne sert d'ailleurs pas à grand chose. Nous pourrons dans un calcul approché risquer une simplification qui est la suivante :

Quand la charge est appliquée en S', elle produit en toute section une influence qui diffère, à un terme variable près, de l'influence que produirait l'application de la charge en $\mathrm{S}$, car les diagrammes de moment diffèrent dans les deux cas d'une constante (fig. 1).

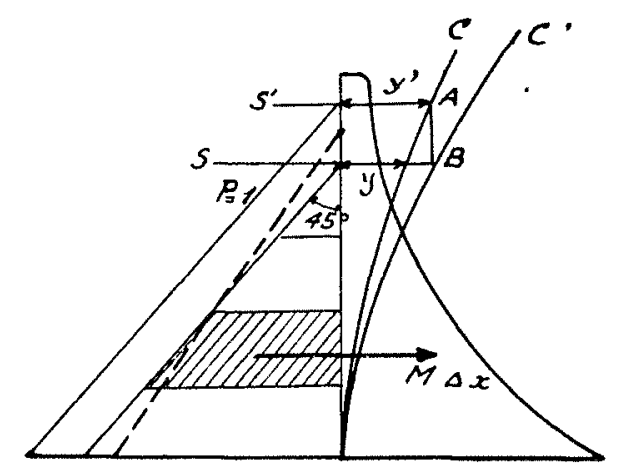

Fig. 1

Mais l'introduction de ce terme variable ne permel pas de trouver une simplification imporlante dans les calculs qui suivront.

Cette façon de voir n'est d'ailleurs pas exacte elle-mênic.

Par contre, supposons que l'on admetie, au lieu de le diflérence de moments, le rapport de moments constant sur loutela hauteur, il en résulte de suite que les ordonnées des deux lignes d'influcnee C, C' sont proportionnelles et daus le mapport $\frac{y}{y^{\prime}}$.

Cette façon de voir semble à priori risquéc et même absurde, mais en fait elle ne diffère beaucoup de la vérité que pour les sections éloignées du sommet pour lesquelles les ordonnées des 


\section{LA HOUILLE BLANCHE}

lignes d'influence sont de plus en plus petites. Ce sont donc les lermes les plus petits qui sont entachés de la plus grande parl d'erreur et l'influence d'ensemble sur les résultats est moindre qu'il ne semble.

Nous n'indiquerons d'ailleurs cette méthode que pour nous en servir dans les calculs de première approximation.

Dans les calculs définitif's, nous prendrons les lignes d'influence une à une, sans chercher à établir de relations entre leurs ordonnées.

Colte parenthèse close, nous voyons qu'il est facile d'obtenir une ou plusieurs lignes d'influence pour les éléments murs.

pour les éléments arcs, il n'en est pas de même. On peut évidemment déterminer par points la ligne des défocmations produites dans l'arc par l'applicalion d'une charge normale à l'arc appliqué en un point. C'est une opération des plus longues.

Supposons toutefois que l'on ait déterminé les lignes d'influcnce relatives aux différents éléments du demi arc. L.es autres

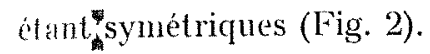

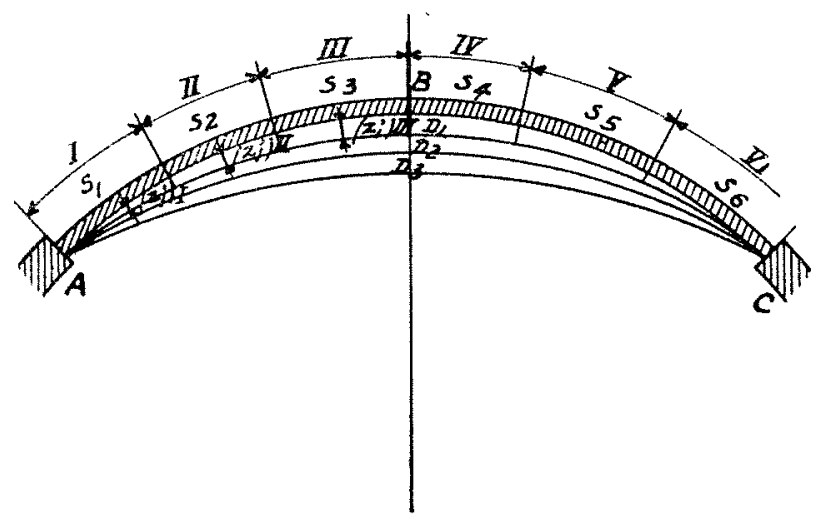

[iig. 2

Nous appellerons

$$
\left|z_{1}^{1}\right|_{1},\left|z_{2}^{1}\right|_{1},\left|z_{3}^{1}\right|_{1}
$$

les ordonnées de la ligne $D_{1}$ suivant les sections $S_{1}, S_{2}, S_{3}$

$$
\left|z_{1}^{2}\right|_{1},\left|z_{2}^{2}\right|_{1},\left|z_{3}^{2}\right|_{1}
$$

celles de la ligne $\mathrm{D}_{2}$ suivant les mêmes sections.

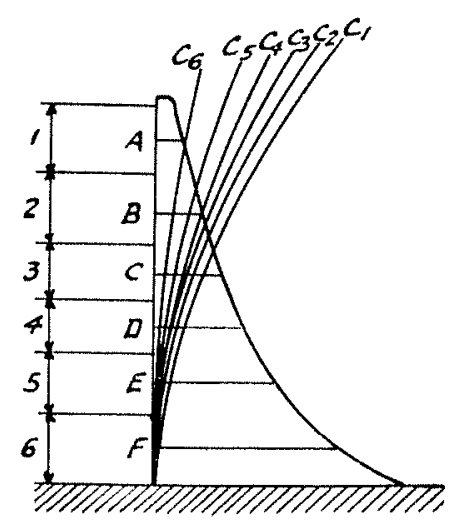

Fig. 3

Considérons de même les lignes d'influence de l'élément mur correspondant à la $1^{\text {re }}$ tranche de l'arc (fig. 3). Le 1/2 arc est divisé en 6 tranches, $\mathrm{C}_{1}$ est la ligne relative à la section médiane de l'élément (1), etc...

Nous représentons par

$$
\left(y_{1}^{1}\right)_{1},\left(y_{2}^{1}\right)_{1},\left(y_{1}^{1}\right)_{1}
$$

les ordonnées de $C_{q}$ suivant les sections $A . B$. C. D.

$$
\left(y_{1}^{2}\right)_{1}, \quad\left(y^{2}\right)_{1}, \quad\left(y_{3}^{2}\right)_{1}
$$

celles de $\mathrm{C}_{2}$ suivant ces sections.

Appelons $K_{1}^{1}, K_{2}^{1}, K_{3}^{1}$ les coefficients de poussćc supportée par le mur suivant ces éléments et $x_{1}, x_{2}, x_{3}$ les distances des sections A B C ău plan supérieur d'eau.

Pour l'élément mur correspondant à la tranche II de l'arc, les lignes d'influence sont changées, les ordonnées des lignes d'influence deviennent

$$
\left(y_{1}^{1}\right)_{\mathrm{r}},\left(y_{2}^{1}\right)_{\mathrm{II}},\left(y_{1}^{2}\right)_{\mathrm{II}},\left(y_{2}^{2}\right)_{\mathrm{II}} \text {, elc. }
$$

Le raisonnement fait sur les tranches de murs se reprend pour les tranches d'arc.

Les ordonnées des lignes d"influence du $2^{\mathrm{e}}$ arc correspondant à la tranche 2 des murs prennent la forme

$$
\left(z_{1}^{1}\right)_{2},\left(z_{2}^{4}\right)_{2},\left(z_{3}^{4}\right)_{2}
$$

Ces notations étant indiquées, écrivons l'égalité des flèches en tout point.

La flèche de l'élément 1 du mur appartenant à l'arc I est d'après une propriété connue des lignes d'influence

$$
f_{1}=\Leftrightarrow\left[\mathrm{K}_{1}^{1} x_{1}\left(y_{1}^{1}\right)_{1}+\mathrm{K}_{2}^{1} x_{2}\left(y_{2}^{1}\right)_{1}+\ldots \mathrm{K}_{6}^{1} x_{6}\left(y_{6}^{1}\right)_{1}\right]
$$

La flèche du même élément considéré comme appartenant à l'arc est

$f_{1}=\omega x_{1}\left\lceil\left(1-K_{1}^{1}\right)\left(z_{1}^{1}\right)_{1}+\left(1-K_{1}^{2}\right)\left(z_{2}^{1}\right)_{1}+\ldots\left(1-K_{1}^{(1)}\right)\left(z_{0}^{1}\right)_{1}\right\rceil$

D'où l'équation

$\mathrm{K}_{1}^{1} x_{1}\left(y_{1}^{1}\right)_{1}+\mathrm{K}_{2}^{1} x_{2}\left(y_{2}^{1}\right)_{1}+\ldots=x_{1}\left[\left(1-\mathrm{K}_{1}^{1}\right)\left(z_{1}^{\mathrm{b}}\right)_{1}+\left(1-\mathrm{K}_{1}^{\mathrm{w}}\right)\left(z_{2}^{1}\right)_{1}+-\right]$

En écrivant des équations semblables pour tous les 6 éléments de chacun des trois murs I, II; III on aura 18 équations entre les 36 coefficients

$$
K_{1}^{1}, K_{\frac{1}{2}}^{\frac{1}{2}} \quad K_{1}^{2}, K_{2}^{\prime \prime} \quad K_{1}^{1}, K_{2}^{\prime !}
$$

qui sont égaux deux à deux, car

$$
\mathrm{K}_{1}^{\mathrm{i}}=\mathrm{K}_{1}^{6}, \mathrm{~K}_{1}^{2}=\mathrm{K}_{1}^{\overline{3}}
$$

Le problème ęst soluble théoriquement, mais pas pratiquement. On peut certainement décomposer en un nombre moindre d'éléments, par exemple le $1 / 2$ arc en deux murs sur chacun desquels on envisagera trois éléments. On aura par la résolution de 6 équations les 6 inconmues représentant les coefficients de poussée moyenue sur chacun des panneaux du barrage. On pourra ensuile étudier chacun de ceux-ci en détail. Mais la méthode perd la moitié de sa valeur d'exactitude si on considère peu d'éléments.

Nous n'indiquerons donc celte première méthode toute théorique que pour en faire sentir la généralité complète, en mème temps, malheureusement, que toute la complexité. 


\section{MÉTHOde APPROCHEF.}

Dans la mélhode que nous allons "exposer maintenant, nous supposerons toujours l'arc divisé en $n$ tranches élémentaires constituant autant de murs que nous décomposerons également en tranches horizontales.

Nous supposerons donc que la poussée est uniforme suivant toute la longueur de larc en corrigeant ensuile les déformations par l'introduction d'une poussée moyenne et par le calcul graphique des flèches exactes.

Nous supposerons de même l'arc à épaisseur constante. Comme il ne l'est généralement pas, $e$ épaisseur, sera la moyenne entre les deux $e$ extrêmes à la clé et aux naissances.

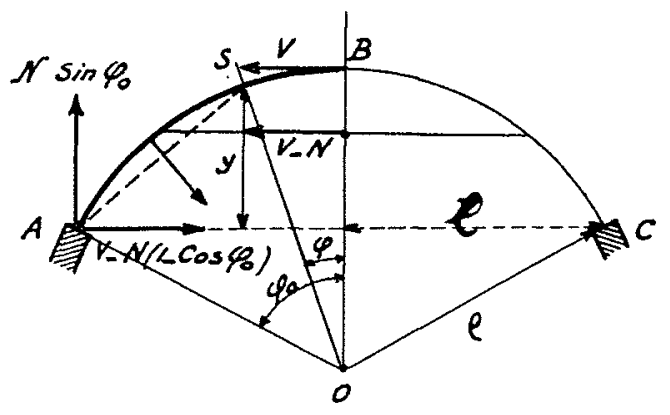

Fig. 4

Calcul des efforts. - Avant d'appliquer la méthode, il est indispensable de reprendre la théorie de l'arc élastique encastré aux extrémités et à courbure constante, qui peut être forte.

Nous supposerons la ligne moyenne parfaitement circulaire d'abord, quitte à la modifier légèrement ensuite (fig. 4).

Nous appellerons $\varlimsup_{0}$ le $1 / 2$ angle d'ouverture de l'arc, $=$ l'abscisse angulaire d'une section quelconque S. Si l'arc était libre aux extrémités, il se développerait simplement un effort de compression $\mathrm{N}=p_{\rho}(p=$ pression, $s=$ rayon $)$ comme dans une conduite cylindrique sous pression.

L'encastrement fait qu'il n'en est pas de même.

Soit donc $V$ la poussée à la clé.

La poussée horizontale en A est naturellement diminuée de la résultante des pressions agissant sur l'arc $\mathrm{AB}$ perpendiculairement à $\mathrm{O} y$.

Cette résultante est :

$$
p p\left(1-\cos i_{0}\right)=N\left(1-\cos \varsigma_{0}\right)
$$

La poussée en A est donc :

$$
V-N(1-\cos \%)
$$

Le moment d'encastrement sera représentée par $M_{1}$, Calculons le moment en $\mathrm{S}$.

Le moment des pressions agissant sur l'arc $\widehat{\mathrm{AS}}$ est :

$$
-p_{\hat{r}} 2 \rho \sin ^{2} \frac{\%-\eta}{2}=-N_{i}[1-\cos (\%-\xi)]
$$

On a donc:

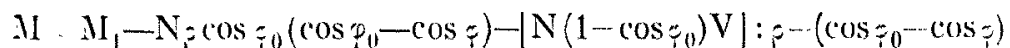

$$
M=M_{1}-(N-V)_{i}\left(\cos \theta_{0}-\cos i\right)
$$

Pour calculer le moment d'encastrement, exprimons que la rotation des sections extrêmes $A$ et $B$ est nulle, ce qui donne:

$$
\int_{0}^{\cdot \pi} \frac{M d s}{\mathrm{E} I}=0
$$

soit :

$$
\int_{0}^{\because \% 0} \mathrm{Md} l_{t}=0
$$

On a :

$$
\mathrm{M}_{1} \xi_{0}-\left(\mathrm{N}^{+}-\mathrm{V}\right){ }_{i}\left(\xi_{1} \cos \xi_{\theta}-\sin \xi_{0}\right)=0
$$

soit :

$$
M_{1}=-(N-V)=\left[\frac{\sin i_{0}}{\psi_{0}}-\cos \approx_{0}\right]
$$

En portant cette valeur dans $M$, il vient:

$$
M=-(V-N) p\left[\cos z-\frac{\sin \xi_{0}}{\sigma_{0}}\right]
$$

Or $\div \frac{\sin \vartheta_{0}}{\varphi_{0}}$ est l'ordomnée $O G$ du centre de gravité de l'arc, $\because \cos F$ est l'ordonnée de la section et on voit que le moment en chaque point est le même que s'il était produit par un effort horizontal $(\mathrm{N}-\mathrm{V})$ appliqué en $\mathrm{G}$. Cet effort agit comme une traction à clé. Si l'arc n'est pas à épaisseur uniforme, G est le centre de gravité élastique, c'est-à-dire le centre des poids élémentaires $\frac{d s}{\mathrm{I}}$. I étant le moment d'inerlie.

Pour calculer $V$, il suffit d'exprimer que l'appui $\Lambda$ est fixe, ou encore mieux, pour tenir compte de suite des actions thermiques qu'il se déplace d'une longueur $x \circ l$.

$\approx$ est le coefficient de dilatation.

0) la variation de température.

$l$ la $1 / 2$ corde de l'arc.

Le déplacement horizonlal produit en A par la llexion est;

$$
\frac{1}{\mathrm{EI}} \int_{0}^{*} \stackrel{*}{\sigma_{0}} \mathrm{I}_{0} y d \tau
$$

qui tend à allonger l'arc. L'effort de compression en $\mathrm{S} a$ a pour composante horizontale $\mathrm{V}-\mathrm{N}(1-\cos \%)$.

Sa valeur est donc :

$$
\frac{V-N(1-\cos i}{\cos i}=N+\frac{V-N}{\cos i}
$$

Le déplacement produit par la compression est :

$$
\frac{1}{\mathrm{E}(2)} \int\left(\mathrm{N}+\frac{\mathrm{V}-\mathrm{N}}{\cos ?}\right) y d
$$

C'est un racourcissement. 
On a donc :

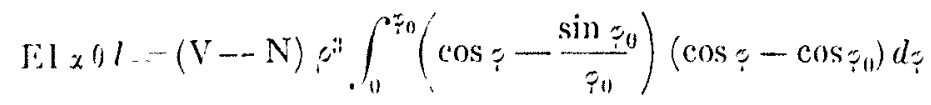

$+\frac{N e^{2}}{12} \% \int_{0}^{\% \pi}\left(\cos \varphi-\cos \tau_{0}\right) d r+\frac{e^{2}}{12}(\mathrm{~V}-\mathrm{N})=\int_{0}^{\% 0}\left(1-\frac{\cos \tau_{0}}{\cos \%}\right) d \%$

Les intégrales sont laciles à calculer :

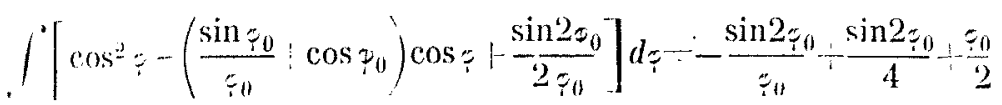

$\int\left(\cos \%-\cos \xi_{0}\right) d \psi=\sin \xi_{0}-z_{0} \cos z_{0}$

$\int\left(1-\frac{\cos \gamma_{0}}{\cos \%}\right) d \%=\tau_{0}-\cos \%_{0} \mathrm{I} \frac{1+\operatorname{tg} \frac{5_{0}}{2}}{1-\operatorname{tg} \frac{\%_{0}}{2}}$

1) oil:

$(\mathrm{V}-\mathrm{N})\left[\mathrm{F}^{2}\left(-\frac{\sin ^{2} \gamma_{0}}{\xi_{0}}+\frac{\sin 2 \gamma_{0}}{4}+\frac{\tau_{0}}{2}+\frac{e^{2}}{12}\left(\xi_{0}-\cos \tau_{0} 1, \frac{1+\operatorname{tg} \frac{\xi_{0}}{2}}{1-\lg \frac{\tau_{0}}{2}}\right)\right.\right.$

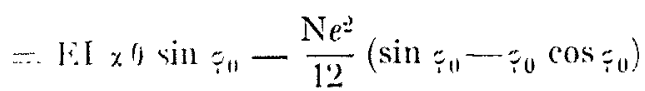

On tire de là :

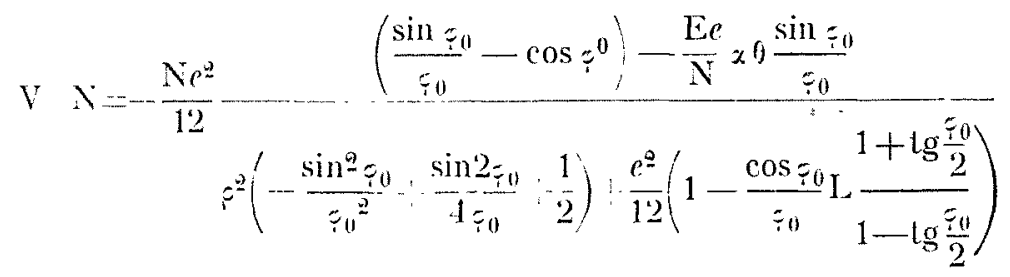

Pour simplifier cette formule, nous allons développer en série les lignes trigonométriques en remarquant que $\tau_{0}$ étant $<1$ radian en général, les termes d'ordre supérieur sont négligeables.

On a ainsi :

$$
\begin{aligned}
& \frac{\sin 7_{0}}{7_{0}}-\cos \gamma_{0}=\left(1-\frac{7_{0}^{2}}{6}\right)-\left(1-\frac{7_{0}^{2}}{2}\right)=\frac{7_{0}^{2}}{3}
\end{aligned}
$$

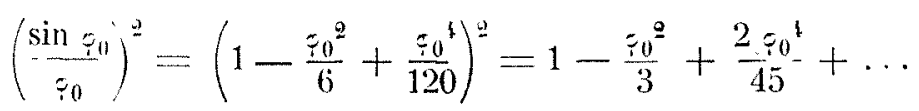

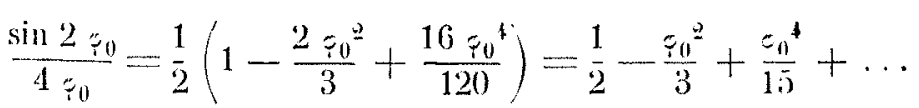

J)'où :

$$
\left.-\left(\frac{\sin \xi_{0}}{\tau_{0}}\right)^{2}+\frac{\sin 2 \tau_{0}}{1}+\frac{1}{2}\right)=\frac{\tau_{0}^{4}}{45}+. .
$$

1) mème

$$
\text { I. } \frac{1+\lg \frac{\sigma_{0}}{2}}{1-\lg \frac{F_{0}}{2}}=2\left(\lg \frac{F_{0}}{2}+\frac{\lg ^{3} \frac{\sigma_{0}}{2}}{3}+\frac{\lg \frac{\sigma_{0}}{2}}{5}\right)
$$

Or:

$\operatorname{tg} \frac{30}{2}=\frac{F 0}{2}+\frac{70^{3}}{24}$

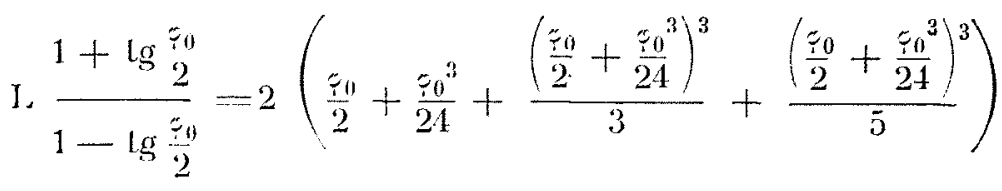

$$
\begin{aligned}
& =7_{0}+\frac{7_{0}^{3}}{6}+\frac{70^{5}}{30}+\ldots
\end{aligned}
$$

D'où :

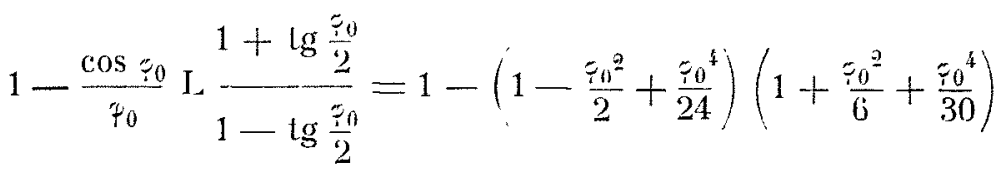

$$
\begin{aligned}
& =\frac{7_{0}^{2}}{3}-\frac{70^{4}}{120}
\end{aligned}
$$

On peut prendre simplement : $\frac{\tilde{z 0}_{0}^{2}}{3}$

On a donc finalement:

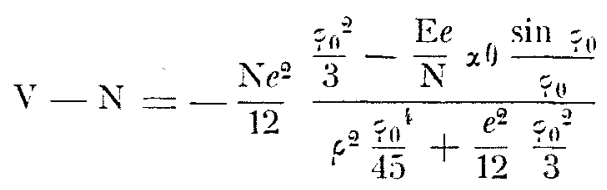

Ou mème en multipliant haut et bas par 36

$$
\mathrm{V}-\mathrm{N}=-\mathrm{N} e^{2} \cdot \frac{\left.\mathrm{F}^{2}\right)^{2}-\frac{3 \mathrm{E} e}{\mathrm{~N}} \times 0 \frac{l}{s}}{0,8 r^{2} 7^{2}+e^{2} e_{10}^{2}}
$$

$\frac{l}{s}$ est le rapport de la corde à l'arc.

Une élévation de température diminue $\mathrm{V}-\mathrm{N}$, donc les moments, par contre un abaissement de température les augmente.

De toutes façons, en appelant $(\mathrm{V}-\mathrm{N})_{0}$ la poussée à la température initiale et $(\mathrm{V}-\mathrm{N})_{0}$ le poussée pour une variation 0 de température. On a :

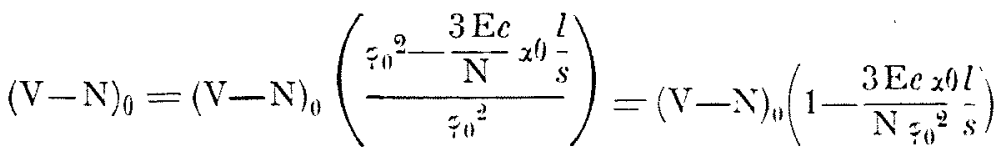

$$
\begin{aligned}
& \text { Le facteur } \\
& \lambda=\left(1-\frac{\left.3 \mathrm{E}_{x}\right)}{30^{2}} \frac{l}{s}\right)
\end{aligned}
$$

où l'on représente par = le travail moléculaire $\frac{N}{e}$ produit à la compression simple, est un facteur qui multiplie les moments. On voit de suite l'influence considérable de ce facteur car $\mathrm{E}_{x}=200.000 \times 0,00001=2 \mathrm{klg} \mathrm{cm} 2$ pour la maconnerie.

Une variation de $20^{\circ}$ donne $\mathrm{E}^{\theta}{ }^{\theta}=40 \mathrm{klgcm}{ }^{2}$ qui dépasse $\overrightarrow{\mathrm{de}}$ beaucoup la valeur admise pour :.

(A suivre). 